\title{
En Bloc Resection of Desmoplastic Neurotropic Melanoma with Perineural Invasion of the Intracranial Trigeminal and Intraparotid Facial Nerve: Case Report and Review of the Literature
}

\author{
Serkan Erkan ${ }^{1}$ Aanand N. Acharya ${ }^{1}$ James Savundra ${ }^{2}$ Stephen B. Lewis ${ }^{3}$ Gunesh P. Rajan ${ }^{1,4}$
}

${ }^{1}$ Department of Otolaryngology, Head and Surgery, Fiona Stanley Hospital, Murdoch, Western Australia, Australia

${ }^{2}$ Department of Plastics Surgery, Royal Perth Hospital, Perth, Western Australia, Australia

3 Perth Neurosurgery, Hollywood Medical Centre, Nedlands, Western Australia, Australia

${ }^{4}$ Skull Base Division, Otolaryngology, Head and Neck Surgery, University of Western Australia, Murdoch, Western Australia, Australia

J Neurol Surg Rep 2016;77:e8-e12.

\author{
Address for correspondence Gunesh P. Rajan, MD, DM, FMH, FRACS, \\ Department of Otolaryngology, Head and Neck Surgery, Fiona Stanley \\ Hospital, Murdoch, WA, 6150, Australia \\ (e-mail: Gunesh.Rajan@health.wa.gov.au).
}

\begin{abstract}
Keywords

- desmoplastic neurotropic melanoma

- skull base

- perineural invasion

- trigeminal neuralgia

- facial palsy

Background Desmoplastic neurotropic melanoma (DNM) is a rare, highly malignant, and locally invasive form of cutaneous melanoma with a tendency for perineural invasion (PNI).

Methods We report a case of a 61-year-old man presenting with right-sided trigeminal neuralgia and progressive facial paresis due to the PNI of the intracranial trigeminal nerve and the intraparotid facial nerve from DNM. We also present a review of the literature with six cases of DNM with PNI of the intracranial trigeminal nerve identified. Results The combined transtemporal-infratemporal fossa approach was performed to achieve total en bloc resection of the tumor mass followed by postoperative radiotherapy (PORT). After 24 months of follow-up, the patient remains disease free with no signs of recurrence on magnetic resonance imaging.

Conclusion We recommend the en bloc resection of the tumor mass followed by PORT for the management of DNM with PNI. A high index of suspicion for PNI as a cause of cranial neuropathies is essential for the early detection and treatment of patients with known melanoma.
\end{abstract}

\section{Introduction}

Desmoplastic neurotropic melanoma (DNM) is a rare, highly malignant, and locally invasive form of cutaneous melanoma associated with a high rate of local recurrence and a low risk of distant metastasis. ${ }^{1-8}$ DNM with perineural invasion (PNI) of the intracranial trigeminal nerve is exceedingly rare with only six cases reported in the literature. ${ }^{9-12}$ PNI confers a higher rate of local recurrence, a poorer prognosis, and worse morbidity compared with DNM without PNI. ${ }^{9,10}$ A significant proportion of this morbidity results from neuropathic pain secondary to clinical PNI of the sensory nerves. Treatment of the disease should therefore focus not only on maximizing disease-free survival but also on adequately managing the morbidity of the disease. However, PNI involving multiple cranial nerves with intracranial extension presents unique received

August 12, 2015

accepted

September 14, 2015

published online

November 3, 2015
DOI http://dx.doi.org/

10.1055/s-0035-1566254. ISSN 2193-6358. (c) 2016 Georg Thieme Verlag KG
Stuttgart · New York

License terms

(c) $(1) \$$ 
operative challenges in terms of access, exposure, and the radicality of resection to maintain quality of life (QOL) and minimize surgical morbidity. Technically, it necessitates a multidisciplinary team approach that utilizes combined skull base approaches to achieve total en bloc resection of the specimen because the pathology usually transgresses several regions in the skull base. Williams et $\mathrm{al}^{11}$ classified the PNI along facial and trigeminal nerves into three zones of involvement. Zones were categorized into distal (zone 1), central/ skull base (zone 2), and cisternal (zone 3). ${ }^{9,11}$ Although there is no treatment guideline, current practice for zones 1 and 2 commonly involves surgical resection followed by postoperative radiotherapy (PORT). ${ }^{9,11-13}$ In cases where PNI has been deemed to be unresectable (zone 3), radiotherapy (RT) can offer various degrees of palliation., 12,14,15

We present a rare case of a DNM with PNI along the right trigeminal and intraparotid facial nerves that was treated by en bloc resection using the piezo surgery device and PORT. To the best of our knowledge, this is the first reported case of DNM with concomitant PNI along the facial and trigeminal nerves with intracranial extension to the gasserian ganglion (GG). This case highlights the utility of the combined skull base approaches to facilitate en bloc resection of the tumor mass from the brainstem to the peripheral nerves involved, providing excellent pain control, improved QOL, and limited morbidity.

\section{Case Report}

\section{History and Examination}

A 61-year-old white man with a past history of excision of a malignant melanoma (spindle cell variant) from the right preauricular region 3 years ago was referred by the neurologist to the tertiary ears, nose, throat skull base clinic with a 9-week history of right-sided progressive trigeminal neuralgia and paraesthesia in the distribution of the right mandibular nerve (V3). This was associated with an increasing ipsilateral facial nerve paresis (House-Brackman grade 4). Initially diagnosed with trigeminal neuralgia, the patient received treatment with carbamazepine, with adjunctive opioid analgesia for breakthrough pain. Despite maximal pain therapy, the neuralgia remained poorly controlled and progressed.

The referring neurologist identified paraesthesia and hyperesthesia in the distribution of the right V3 and a right lower motor neuron facial nerve paresis. Mild wasting of the ipsilateral temporalis and masseter muscles was noted. Magnetic resonance imaging (MRI) of the head revealed an extensive perineural tumor in the right masticator space that extended along the branches of V3 medially, posteriorly, and laterally with invasion of the right parotid gland and involvement of the intraparotid divisions of the right facial nerve with anterograde enhancement to the stylomastoid foramen. Superiorly, the tumor extended along the mandibular nerve to the foramen ovale and into the inferior aspect of the GG (-Fig. 1). The lesion was deemed unresectable at a different institution. The patient was subsequently referred to our skull base clinic for a second opinion.

\section{Surgical Procedure}

En bloc resection of the tumor mass was achieved by a combined transtemporal-infratemporal approach. A hemicoronal postauricular incision with extension into the upper neck was performed. A lateral mandibulotomy was performed using the piezo surgery device to increase the access to the infratemporal fossa (ITF) and to access the inferior alveolar nerve. The mental nerve was identified and divided at the mental foramen and pulled proximally through the alveolar canal. Via a cortical mastoidectomy, the facial nerve was skeletonized from the first genu to the stylomastoid foramen, divided at the first genu, and delivered in continuity with the entire parotid component. Then an extended middle cranial fossa craniotomy with a petrous apicectomy was performed to access both the middle and posterior cranial fossae. This access was used to release the trigeminal nerve at the root entry zone with subsequent delivery of the entire trigeminal ganglion in continuity with V3 and the content of the right infratemporal fossa and the right parotid compartment (-Fig. 2). The ophthalmic and maxillary nerves were detached at the level of their respective foramina to mobilize
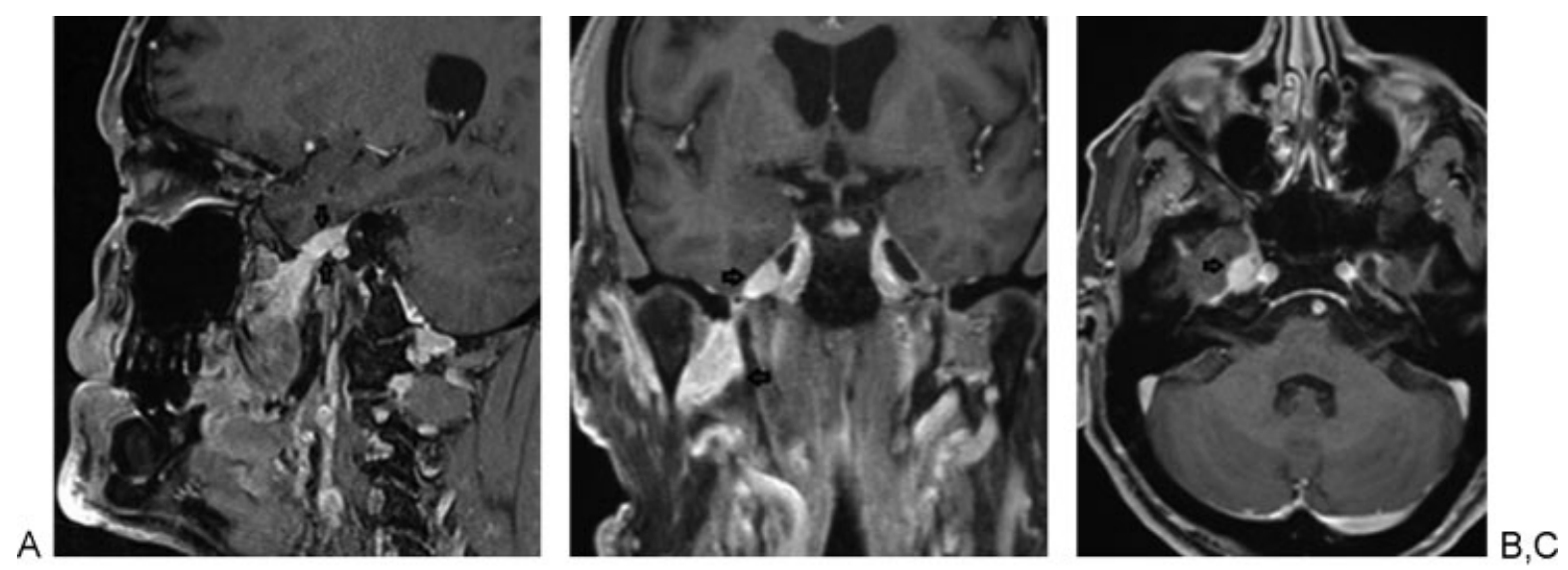

Fig. 1 T1-weighted (A) sagittal, (B) coronal, and (C) transverse magnetic resonance imaging of the head demonstrates contrast enhancement of the trigeminal ganglion, trigeminal nerve (black arrows), and intraparotid divisions of the right facial nerve. 


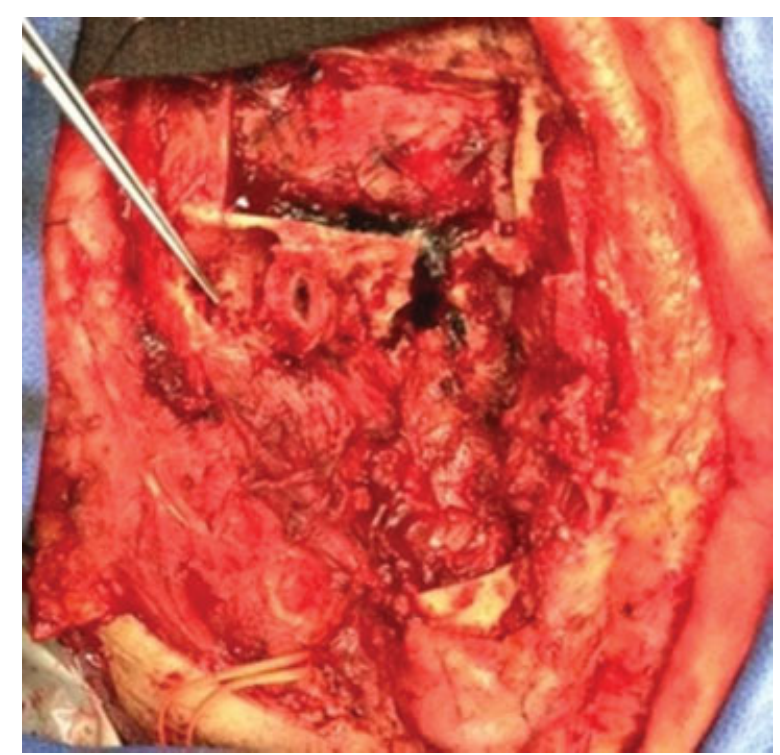

Fig. 2 Situs with the extended middle fossa craniotomy combined with the infratemporal fossa approach after the en bloc removal of the specimen.

the GG, thus enabling the amputated trigeminal nerve, the trigeminal ganglion, and the contents of the ITF to be mobilized and delivered inferiorly in continuity into the upper neck (-Fig. 3). The defect was reconstructed with a gracilis muscle free flap and covered with a split skin graft.

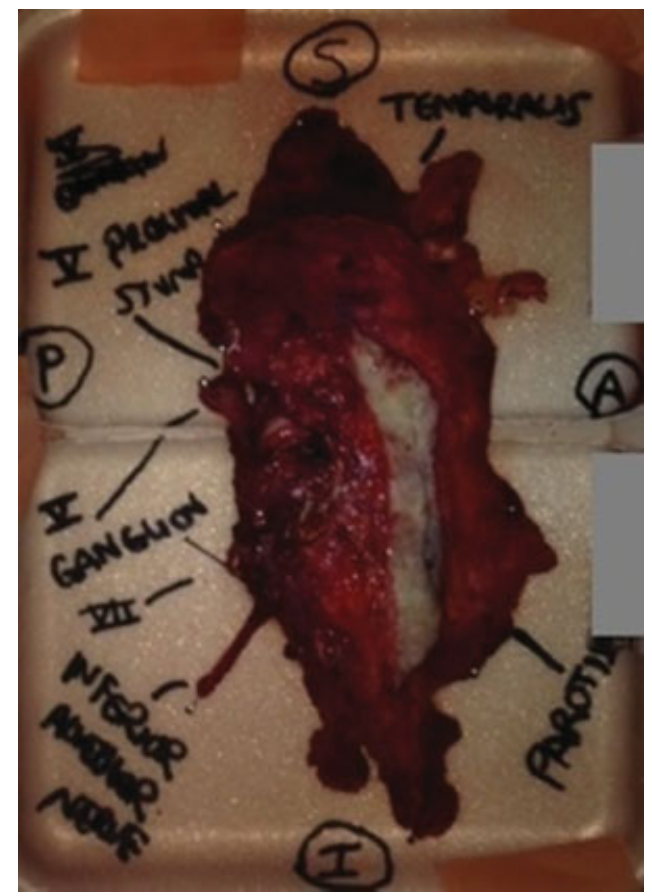

Fig. 3 En bloc resection of the tumor mass shows proximal trigeminal stump in continuity to the inferior alveolar nerve, facial nerve, and parotid gland.

\section{Histopathology}

Histopathologic analysis demonstrated cellular fascicles of spindled cells with marked pleomorphism and abundant mitotic activity. There was a prominent inflammatory and reparative response surrounding the tumor as a sign of ongoing immunologic response. Immunohistochemical analysis revealed patchy positive staining of tumor cells with melanoma markers S100, tyrosinase, and microphthalmiaassociated transcription factor (MITF). The tumor cells were negative for other melanoma markers HMB45 and Melan A. This was in keeping with DNM. The resection margins peripherally along the inferior alveolar nerve up to the mental nerve and the branches of the facial nerve were clear. Centrally, the perineural invasion involved the entire GG. Zone 3 and the proximal trigeminal root entry stump were free of PNI. No skip lesions were present.

\section{Postoperative Course}

The patient was discharged and underwent a course of postoperative intensity-modulated RT, $60 \mathrm{~Gy}$ in 30 fractions. This was complicated by the development of an orocutaneous fistula from the site of the lateral mandibulotomy to the incision line in the neck. It was successfully managed with intravenous antibiotics and hyperbaric oxygen. The facial nerve palsy was managed with static techniques and appropriate eye care. The follow-up imaging revealed a pseudomeningocele in the superior ITF measuring $50 \times 48 \times 50 \mathrm{~mm}$. This spontaneously reduced in size on further imaging follow-up measuring $33 \times 35 \times 38 \mathrm{~mm}$ (-Fig. 4). After 24 months of follow-up, the patient remains disease free with no signs of recurrence clinically and on MRI imaging. The patient has recovered well with complete pain resolution since surgery. His vision is unaffected. He recommenced employment 6 months after completion of the RT and has good QOL.

\section{Discussion}

DNM was first described by Reed and Leonard in 1979 as a subtype of desmoplastic melanoma exhibiting neurotropism. ${ }^{16}$ The immunochemical diagnosis is based on positive melanoma markers that include S100, tyrosinase, and MITF, and it is negative for other markers such as HMB45 and Melan A. ${ }^{17}$ However, more recently, P75 NGF-R has been proposed as a superior diagnostic stain to $\mathrm{S} 100 .^{18}$

Since 1979, several case series have confirmed that DNM has a significant risk of PNI, a poor prognosis, and local recurrence compared with other malignant melanomas. ${ }^{1,2,6-9,13,19}$ DNM clinically manifests as an amelanotic nodule or scar-like lesion that can often make diagnosis difficult. ${ }^{19,20}$ Quinn et $\mathrm{al}^{4}$ found that the local recurrence was higher if the surgical margin was $<1 \mathrm{~cm}$. However, there was no improvement in recurrence rate where margins were $>2 \mathrm{~cm}$. The optimum margin therefore appears to be a minimum of $1 \mathrm{~cm}$. There is also evidence for the use of PORT in cases of PNI. Guadagnolo et $\mathrm{a}^{15}$ found the local recurrence rate to be higher in patients treated with surgery only (24\%) than in those treated with surgery and postoperative RT (7\%). 

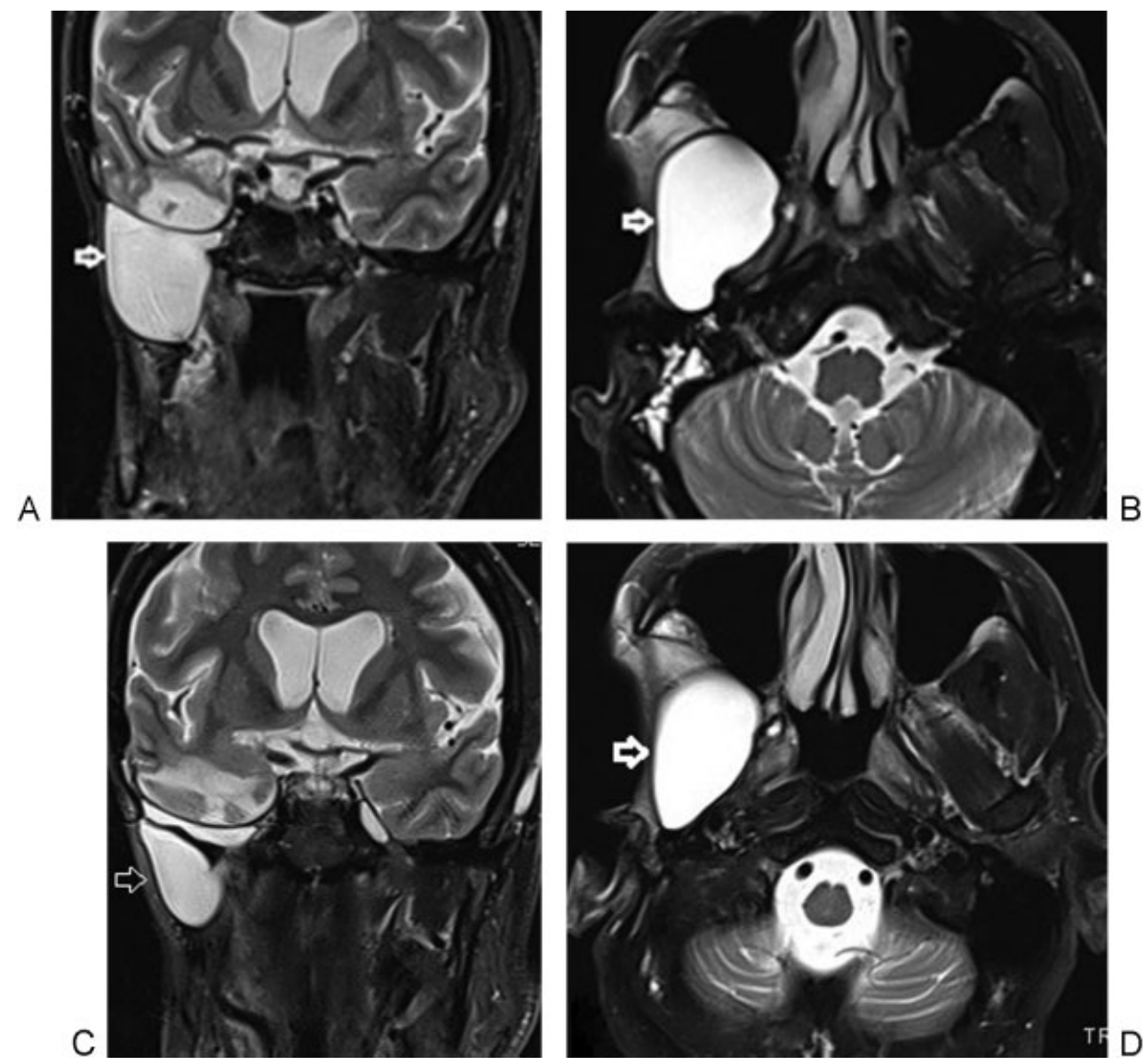

Fig. 4 Postoperative magnetic resonance imaging performed at (A, B) 9 months and (C, D) 18 months shows pseudomeningocele (arrows) reducing in size.

Similarly, Strom et $\mathrm{al}^{22}$ also found that adjuvant RT was associated with better local control in patient with both positive and negative resection margins. The rate of local recurrence for patients with positive resection margins was only $14 \%$ with PORT compared with a $54 \%$ recurrence rate for those who did not receive PORT. ${ }^{22}$

Only six cases have been reported in the published literature that describe the management of DNM with PNI involving the intracranial part of the trigeminal nerve. . $^{21,23,24}$ Walters et al performed total resection of the tumor involving the GG using the supra- and infratemporal fossa approach followed by PORT. ${ }^{20}$ In another case, a piecemeal subtotal resection was performed for a mass filling the Meckel cave, with residual tumor left in situ with the postoperative histology showing DNM. The patient received PORT. However, the residual tumor was noted to have progressed on a 5 -month follow-up MRI scan. ${ }^{23}$ Lin et al ${ }^{21}$ described a case of DNM with trigeminal nerve involvement extending to the skull base. Definitive RT was the treatment of choice. The patient was disease free after 10 months of follow-up. Newlin et $\mathrm{al}^{24}$ described their experience of three cases of DNM with PNI. Definitive RT was the treatment of choice for two patients, one of whom demonstrated tumor proximal to the trigeminal ganglion and the other of whom had tumor bulk demonstrable at the root of the trigeminal nerve in the brainstem. In the first case, the patient had a disease-free survival of 34 months after treatment with RT but experienced significant ocular complications from the treatment. In the second case, the patient developed a recurrence in a right buccal node after 2.5 years that required salvage surgery. In the third case, the patient underwent resection of the trigeminal nerve at the level of the root entry, with residual tumor in the pons that was treated with RT. This was complicated by temporal lobe necrosis, necessitating temporal lobectomy. The patient was disease free 14 months after treatment. ${ }^{24}$

DNM with PNI is associated with a low rate of distant metastases. ${ }^{1-5}$ Consequently, the disease warrants an aggressive approach to treatment if long-term local control is to be achieved. ${ }^{9,25}$ Furthermore, en bloc resection effectively eliminates PNI-related trigeminal neuropathic pain in disease involving zones 1 and 2 . Thus the en bloc resection of such tumors, including the trigeminal ganglion and proximal trigeminal nerve, maximizes local tumor control and symptom control and minimizes the risk of local recurrence of disease. ${ }^{9,12,26}$ The combined transtemporal-infratemporal fossa approach facilitates adequate access and exposure without any active brain retraction for the en bloc resection of the tumor involving the trigeminal and facial nerves without postoperative surgical morbidity or a decrease in 
QOL. The introduction of piezo surgery further enables the combination of various approaches without the risk of injury to critical neurovascular structures such as the adjacent cranial nerves, internal carotid artery, or venous sinus in the skull base. ${ }^{27,28}$

It is noteworthy that surgical intervention changes the natural progression of DNM with PNI. Without treatment or in recurrence after RT, the disease eventually infiltrates the brainstem with subsequent death within a few months. The surgical treatment prevents proximal central progression of disease. However, it can result in peripheral recurrences after surgery that are usually in the skin, often with multifocal distribution at the resection margins or the borders of the radiation treatment. These recurrences are very challenging because surgically there are limitations on how much skin can be resected in the face and the head and neck.

\section{Conclusion}

DNM with PNI is a rare but aggressive tumor with a high rate of local recurrence, a poor prognosis, and an adverse functional outcome. However, the low rate of metastatic spread means that chances of effective local control can be maximized by an aggressive approach to treatment. Combined skull base approaches afford adequate access and exposure for the en bloc resection of PNI with intracranial extension involving both the trigeminal and facial nerve. Hence en bloc resection with PORT is recommended with the benefits of minimizing the risk of iatrogenic tumor dissemination, local recurrence of disease, and maximizing control of pain symptoms and disease-free survival. Clinicians should have a high level of suspicion for PNI as a cause of cranial neuropathies in patients with a known history of melanoma or other cutaneous malignancies such as squamous cell carcinoma and basal cell carcinoma located in the head and neck region. Early detection and treatment increase disease-free survival with preservation of QOL.

\section{References}

1 Chen LL, Jaimes N, Barker CA, Busam KJ, Marghoob AA. Desmoplastic melanoma: a review. J Am Acad Dermatol 2013;68(5): 825-833

2 Chen JY, Hruby G, Scolyer RA, et al. Desmoplastic neurotropic melanoma: a clinicopathologic analysis of 128 cases. Cancer 2008; 113(10):2770-2778

3 Anstey A, McKee P, Jones EW. Desmoplastic malignant melanoma: a clinicopathological study of 25 cases. Br J Dermatol 1993;129(4): 359-371

4 Quinn MJ, Crotty KA, Thompson JF, Coates AS, O'Brien CJ, McCarthy WH. Desmoplastic and desmoplastic neurotropic melanoma: experience with 280 patients. Cancer 1998;83(6): 1128-1135

5 Murali R, Shaw HM, Lai K, et al. Prognostic factors in cutaneous desmoplastic melanoma: a study of 252 patients. Cancer 2010; 116(17):4130-4138

6 Jain S, Allen PW. Desmoplastic malignant melanoma and its variants. A study of 45 cases. Am J Surg Pathol 1989;13(5): 358-373
7 Carlson JA, Egbert BM. Desmoplastic neurotropic malignant melanoma. Pathology (Phila) 1994;2(2):339-357

8 Carlson JA, Dickersin GR, Sober AJ, Barnhill RL. Desmoplastic neurotropic melanoma. A clinicopathologic analysis of 28 cases. Cancer 1995;75(2):478-494

9 Panizza B, Warren T. Perineural invasion of head and neck skin cancer: diagnostic and therapeutic implications. Curr Oncol Rep 2013;15(2):128-133

10 Johnston M, Yu E, Kim J. Perineural invasion and spread in head and neck cancer. Expert Rev Anticancer Ther 2012;12(3): 359-371

11 Williams LS, Mancuso AA, Mendenhall WM. Perineural spread of cutaneous squamous and basal cell carcinoma: CT and MR detection and its impact on patient management and prognosis. Int $\mathrm{J}$ Radiat Oncol Biol Phys 2001;49(4):1061-1069

12 Anderson TD, Weber RS, Guerry D, et al. Desmoplastic neurotropic melanoma of the head and neck: the role of radiation therapy. Head Neck 2002;24(12):1068-1071

13 Panizza B, Solares CA, Redmond M, Parmar P, O’Rourke P. Surgical resection for clinical perineural invasion from cutaneous squamous cell carcinoma of the head and neck. Head Neck 2012; 34(11):1622-1627

14 Barnett CM, Foote MC, Panizza B. Cutaneous head and neck malignancies with perineural spread to contralateral cranial nerves: an argument for extending postoperative radiotherapy volume. J Clin Oncol 2013;31(18):e291-e293

15 Guadagnolo BA, Prieto V, Weber R, Ross MI, Zagars GK. The role of adjuvant radiotherapy in the local management of desmoplastic melanoma. Cancer 2014;120(9):1361-1368

16 Reed RJ, Leonard DD. Neurotropic melanoma. A variant of desmoplastic melanoma. Am J Surg Pathol 1979;3(4):301-311

17 Banerjee SS, Harris M. Morphological and immunophenotypic variations in malignant melanoma. Histopathology 2000;36(5): 387-402

18 Lazova R, Tantcheva-Poor I, Sigal AC. P75 nerve growth factor receptor staining is superior to $\mathrm{S} 100$ in identifying spindle cell and desmoplastic melanoma. J Am Acad Dermatol 2010;63(5): 852-858

19 Moonis G, Cunnane MB, Emerick K, Curtin H. Patterns of perineural tumor spread in head and neck cancer. Magn Reson Imaging Clin N Am 2012;20(3):435-446

20 Walters H, Lewis E, Wolper R, Yachnis AT, Green J, Lewis S. Neurotropic melanoma of the trigeminal nerve: a case of atypical facial pain. J Oral Maxillofac Surg 2008;66(3):547-550

21 Lin D, Kashani-Sabet M, McCalmont T, Singer MI. Neurotropic melanoma invading the inferior alveolar nerve. J Am Acad Dermatol 2005;53(2, Suppl 1):S120-S122

22 Strom T, Caudell JJ, Han D, et al. Radiotherapy influences local control in patients with desmoplastic melanoma. Cancer 2014; 120(9):1369-1378

23 Hashemi M, Stark A, Hugo H, Mehdorn M. Intracranial trigeminal nerve metastasis of a desmoplastic neurotropic melanoma: case report. Cent Eur Neurosurg 2009;70(2):91-94

24 Newlin HE, Morris CG, Amdur RJ, Mendenhall WM. Neurotropic melanoma of the head and neck with clinical perineural invasion. Am J Clin Oncol 2005;28(4):399-402

25 Raizer JJ, Hwu WJ, Panageas KS, et al. Brain and leptomeningeal metastases from cutaneous melanoma: survival outcomes based on clinical features. Neuro Oncol 2008;10(2):199-207

26 Foote MC, Burmeister B, Burmeister E, Bayley G, Smithers BM. Desmoplastic melanoma: the role of radiotherapy in improving local control. ANZ J Surg 2008;78(4):273-276

27 Kotrikova B, Wirtz R, Krempien R, et al. Piezosurgery-a new safe technique in cranial osteoplasty? Int J Oral Maxillofac Surg 2006; 35(5):461-465

28 Beziat JL, Bera JC, Lavandier B, Gleizal A. Ultrasonic osteotomy as a new technique in craniomaxillofacial surgery. Int J Oral Maxillofac Surg 2007;36(6):493-500 